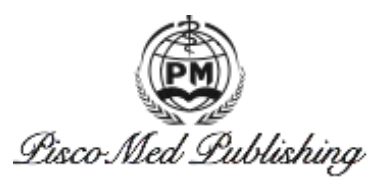

Original Research Article

\title{
Research on Pear Video Information Communication Strategy
} Xin Zhang*

Jincheng College of Sichuan University. E-mail: zhangx@163.com

Abstract: From 2012 to 2015, the development of China's short video industry has been in an exploratory period, with platforms such as Aauto Quicker, beautiful shooting and second shooting. In 2016, the development of short video in China ushered in a growth period, and the number of short video applications increased sharply, which benefited from the improvement of various mobile services, the change of audience reading habits and the capital investment of Internet giants in the short video industry. The competitive advantage of short video is that it is short and pithy, and can make users get rich content in the shortest time, which can meet the needs of users for fragmentation. With the continuous development of short video, short video applications begin to show vertical characteristics, and content production extends to vertical fields, such as food, music, beauty and information. Pear Video was launched in November, 2016, which is positioned to focus on the application of short information video, and it is also an early application of short information video in China. Studying its communication strategy has reference significance for the development of short-sighted information industry.

Keywords: Pear Video; Information; Communication Strategy

At present, the application of short-sighted applications in China mainly focuses on entertainment positioning, such as Aauto Quicker and Mei Pai. Pear video appeared recently in China. There are few short video platforms, and the academic circles pay less attention to them. With the deepening of the verticalization of short videos, the needs of viewers began to show diversified development. Short videos communication strategy has become the most important challenge for the platform creation team.

\section{Information short video}

\subsection{The definition of short video}

As short video is a new thing, there is no clear definition of short video in academic circles. Since short video is called "short" video, compared with long video, short has three meanings. The first is the short duration of video. Long-term vision is more than half an hour. A kind of video produced by professional media or public organizations, including movies, TV dramas, variety shows, documentaries, etc. According to the concept of long video, the criteria for judging whether a video is a long video are more than half an hour and professional content producers. A video that exceeds half an hour is a long video, so the duration of a short video must not exceed 30 minutes. This is also the academic consensus on short video duration. However, at present, most videos on various video websites and short video apps are within 10 minutes, and videos with high clicks are no longer than 5 minutes. Therefore, this paper thinks that the best time for the moment is no more than 5 minutes. Second, the production time is short. As the video duration be-

This is an open-access article distributed under the terms of the Creative Commons Attribution Non-Commercial License (http://creativecommons.org/licenses/by-nc/4.0/), which permits unrestricted non-commercial use, distribution, and reproduction in any medium, provided the original work is properly cited. 
comes shorter, the time required for pre-shooting, post-editing and beautification of the corresponding video will also become shorter. This is also the obvious difference between short video and general video. Third, it takes a short time to spread. The content of the short video is more concise and the form is convenient for the audience to accept. Short videos can be shared in real time on social platforms to meet the fast-food consumption of audiences. Therefore, it can reach a high transmission rate in a short time, and the time consumed by transmission and insertion is naturally shortened.

For the definition of short video, besides the explanation of "short", there is another word "video". The implication of "seeing cheeks" is content. Producers of long videos are professional organizations, namely PGC (Professional Production Content), while producers of short videos can be audience, namely UGC (User Production Content), or professional organizations. In addition, short videos are short in time. Content is concise and easy to share on social platforms. The user's acceptance is also high, so short videos are more social. To sum up, combined with the background of short video, the difference from long video, the characteristics of moment video and so on. This paper argues that short video is a new form of information expression, which can be quickly shot, edited and beautified by professional media or individuals using mobile terminals, and can be shared and interacted on new media platforms and social platforms.

\subsection{Overview of pear video}

Pear video positioning is short-sighted, and its main function positioning is to discover information, transmit information and share information. Therefore, Pear Video is the main short video product of information, and the length of video is mainly between 30 seconds and 3 minutes. Pear video is also the first short video application focusing on information in China. After the transformation, the content of Pear Video shows the characteristics of being out of date and political. At present, the application of pear video has 13 categories: local, new knowledge, society, world, life, science and technology, entertainment, wealth, automobile, food, sports, music and secondary. Users can search and pay attention to related classifications according to their own preferences.

\section{There are problems in Pear Video communication strategy}

\subsection{The pursuit of interesting content depth is low}

In China, the management of short video platform for network new media is constantly improving, and the legitimacy of the transformed Pear Video in news interview is relatively improved. However, after the transformation, the characteristics of Pear Videos in content decline, and the pursuit of interesting information content leads to lower content depth. There are three main reasons.

First, the standard for judging the value of information is lowered. Compared with current political information, social information is more interesting and entertaining, and the value of information becomes lower. Time novelty, importance, distinctiveness, proximity and interest are the five essential elements of high-value news. However, in the information content of the objective Pear Video, the requirements for novelty and importance are reduced, and the significance, proximity and interest are more obvious in its information. Especially interesting, because Pear Video requires interesting and informative content, and it excessively pursues the interest of short video content, which makes the information show a certain state of pan-entertainment.

Second, the content is mainly fragmented. The biggest feature of short video is its short time, close to civilian life and fast transmission speed. The short video content in Pear Video also has such characteristics. Pear Video pays attention to stories and humanized events, and how to combine the quality and depth of monthly content has become the primary problem to be solved. If social topics want to reflect their depth, they need to be used to express the emotional changes and contradictions of characters in social events through deep processing and lens language after obtaining a large number of relevant news clues. However, in the social news information presented by Pear Video, due to the limitation of time, the information content only shows the core part of news events, unable to present complete news content, unable to trace the news process, and even more difficult to show the delicate emotional changes and contradictions of characters. Therefore, the news information selected by Pear Video is the content with simple plot. These 
contents belong to news clues with low content depth and low information value in traditional media news reports. When selecting news content, Pear Video overemphasizes interest and communication arrival rate, and caters to audience blindly, which reduces the depth of content to a certain extent.

Third is secondary media production. The content production of Pear Video mainly uses the platform and the photographer to produce the content together. The content provided by the photographer is audited and edited by the professional team in the later stage, which is called secondary media production. Short-sighted content is provided by the photographer, which can guarantee the platform content source and daily video output. However, social shooters are the main shooters of Pear Videos, while professional shooters and official institutions account for a small proportion. This will reduce the specialization of short video content production to a certain extent. Pear Video mainly focuses on information shortsightedness, and some contents are paid short video products. Since you need to pay, you need the content itself to have a value curve. The publicity of Pear Video mainly emphasizes discovering fresh highlights around us and conveying positive energy of society and kindness of human nature. Most of the news with strong timeliness produced by traditional media is sufficient for one-time consumption, while the content topics selected by Pear Video tend to be secondary consumption content. The production of this kind of information content is suitable for the audience to watch repeatedly, which can cause extensive discussion in the society and reflection from the audience. According to the information short video content produced by Pear Video at present. The content that causes extensive discussion is only because of its interest, not the depth behind its content. Pear Video ignores the depth and value of secondary consumer products when making secondary media of content.

To sum up, due to the lowering of the standard of judging the value of news information, the fragmentation of content expression and the secondary media production, the content of Pear Video information does not have the ability of secondary consumption, and the short video of information shows the deficiency of over-pursuing interest and neglecting depth.

\subsection{Strong external channel communication, low self-owned platform traffic}

Pear Video adopts multi-platform and large-scale distribution mechanism. This network-wide distribution can promote the dissemination of Pear Video content, help it to obtain user data, analyze and tap users' interests and preferences, and further improve content production and distribution. However, the whole network distribution also brings negative effects. The short video produced by Pear Video is distributed to the audience through news information platform, social platform and traditional video website, which brings high video clicks and likes. But these flows belong to the content distribution platform. Compared with watermelon video, fast video and other information short video platforms, Pear Video's own platform has lower traffic.

The second is to focus on being a content producer and over-rely on the whole network for distribution. At present, the creative team of Pear Video is too optimistic about the external content distribution platform, while ignoring the improvement of its own platform channel communication control. Pear Video has been focusing on the production of high-quality content, while ignoring how to dig out the added value of high-quality content to build its own platform and improve its content distribution ability.

And third is to ignore platformization, which refers to the establishment of Pear Video Website. Innovative platform application. Social platforms are highly interactive, and Pear Video pays too much attention to the interaction with social platforms when formulating communication strategies, including content distribution and brand promotion. The main audience of Pear Video is young people, who are the main users of Weibo. When Pear Video formulates positioning strategy and content strategy based on interactive principle, it pays too much attention to interacting with audience through external media platform. As for platformization, Pear Video ignores the perfection of its own platform function except establishing a global filming system.

\subsection{Lack of platform interaction and low audience stickiness}

The videos forwarded by Lishijiao via social media have high audience participation, and the arrival rate, broad- 
cast rate, praise amount and comment amount of short videos are all high. There are often short videos forwarded by mainstream media on Weibo, which can exceed 40 million in one day. All these indicate that Pear Video itself is an interaction between the audience and the audience, but these interactions are limited to social media and external content distribution platforms. In the short video application list released by Cheetah Big Data, Pear Video users are not active, and lack of interaction with audience on the platform leads to low audience stickiness.

\section{Development Countermeasures of Pear Video Communication}

\subsection{Strengthen professional team building to create high-quality content IP}

IP actually refers to the intellectual property rights of high-quality content. China's current management mechanism for short video content is not perfect, and its boundaries are not clear. Pear Video, as a content producer, needs to create high-quality content IP if it wants to occupy a place in short-sighted industry competition. After IP effect, video copyright can be guaranteed, and scarce high-quality content can accumulate a lot of popularity in a short time, realizing content realization quickly, which will promote the development of the platform. What is most needed to build content IP is to have scarce and deep excellent content. At present, the biggest feature of Pear Video is its largest filming system in the world, with more than 30 million photographers covering all cities in China. At present, the main problem in the content production of Pear Video is that it cannot balance the relationship between smallness and depth. Because Pear Video adopts the content production mode of cooperation between photographers and professional editing teams, in order to create high-quality content IP, it is necessary to strengthen the professional ability of photographers and editing teams and improve the content production capacity.

First, strengthen the management and incentive of the filming team. Photographer is the main content source of Pear Video. Photographer collects valuable video content from all over the world, and presents the video content to audience through the review and evaluation of professional team. There are a large number of videos produced by photographers. In addition to ensuring the professionalism of the manual nuclear application team, Pear Video should first strengthen the management of photographers and improve their professional ability. This requires Pear Video to continuously improve the entry barriers for photographers, and strengthen the management of photographer certification and territorial management. All regions regularly train photographers to enhance their ability to distinguish news, and ensure that news interviews are within a legal and reasonable scope and do not violate the policy bottom line. Second. Improve the incentive mechanism of filming customers. In September, 2017, the Volcano Video application under Today's Headline announced that it would spend 1 billion yuan to inspire short video creators. The Volcano Video will distribute the creator's remuneration according to the fire value, and launch the talent cultivation plan, giving the corresponding fire value according to the contribution degree of the content. The firepower value is calculated according to the amount of video viewing and interaction. This greatly stimulates content production. Pear Video can learn from the incentive mode of volcano video to photographers, so as to enhance the production capacity of photographers' news and ensure the enthusiasm and enthusiasm of photographers' content creation. In the massive information content provided by the photographer, it can be ensured the strong content productivity of Pear Video and provide support for building content IP.

Second, adhere to the characteristics of existing content. In terms of content selection, it is important to pay attention to humanity, story and natural social attributes. In the content production, it is necessary to make full use of the post-editing and secondary processing of video, limit the short video duration, and ensure that the video duration is within the time period when the audience can concentrate on watching. At the same time, it is also crucial to pay attention to the richness of content. For example, the place where subtitles are added can be spread from the characters' words to other places in the video, and screenshots are inserted in the key parts of the video for explanation, which makes the event process clearer. In a word, on the basis of not violating the authenticity of news, the short information video has become prominent and widely accepted by users.

At the same time, the angle of topic selection should be innovated and the regionalization should be strengthened. Pear video, which lacks the qualification of current political information interview, turns to social information. Although there are other types of information in the platform, the value of information is low and the perspective is similar 
to that of other platforms. At present, the single theme type of content will reduce the attraction of content and the quality capital of content. To solve this problem, firstly, pear video can enhance content regionalization. Short videos like Aauto Quicker are popular in fourth-and fifth-tier cities. The content on the platform is regional and close to the audience, so there are more users on the platform. Pear Video can enhance the output of local column content and enhance the regionalization of content topic selection in content production. Secondly, it is important to innovated the angle of topic selection, abandon the inherent thinking of traditional media reports, choose different perspectives to show stories with human feelings in life, and change the topic selection of news from focusing on facts to focusing on social experience. Innovation in the field of concern and new perspectives can bring competitive advantages to Pear Video in the era when content is the most important.

Finally, narrative pays attention to the integrity and continuity of events. Most of the short videos' contents are characterized by de-integrity, which means to emphasize the presentation of a certain attractive segment. However, if Pear Video wants to create high-quality content, it must be different from other short video content production standards. It is necessary to present the integrity of news stories through the expression of short videos. Only by ensuring the integrity, can the value of information content be improved and the audience of Pear Video be attracted to participate.

\subsection{Strengthen the sociality of the platform and enhance the stickiness of the audience}

Social platform has its own social attributes, which can meet the social needs of the audience, so the audience is sticky. The low stickiness of pear video audience is largely due to the lack of sociality and interactivity of Pear Video platform. In the era of social media, users' loyalty cannot be fully obtained only by high-quality content. Therefore, Pear Video should enhance the socialization of the platform. Aauto Quicker videos are used as a short social video. User activity is high, and platform socialization is strong. Specifically, there are many platforms and communication communities, and the pages are simple and easy for users to operate. Second, enhance the user experience. Pear Video can use big data technology to enhance the internal platform's big data processing and analysis capabilities. According to the search and viewing records of potential users, it is necessary to recommend personalized content resources for the audience, and pay attention to the advanced nature of the recommended content. To be advanced refers to the depth of content from shallow to deep, just like learning, from basic simple content to complex content. The depth of the content provided has been continuously improved, which is helpful to satisfy the audience in obtaining information. Using artificial intelligence and algorithm technology, fast and accurate practical information content for the audience can be provided at the first time. At the same time, content push is used to help audience form platform usage habits. Thirdly, it is important to establish mutual assistance mechanism between advanced users and ordinary users to improve user loyalty. Users of the platform are classified into advanced users and ordinary users. Advanced users refer to loyal users of the platform, who are skilled in shooting skills and are the core leaders of the community. Ordinary users refer to potential users of Pear Video and new users who have just used the platform. In the traditional news communication, in order to arouse the enthusiasm of the audience and improve the audiences' loyalty, it is necessary for the opinion leaders to output their opinions and enhance the audiences' sense of questioning with their influence. Then, if the social platform wants the audience to actively participate in it, it can also adopt this form.

\subsection{Focus on building its own platform to reduce social media diversion}

The content produced by Pear Video generates huge traffic on social media platforms, but these user traffic belongs to these media platforms that distribute Pear Video content, which is not translated into the platform traffic of Pear Video itself. Pear Video has adopted the form of social media distribution for brand promotion since its inception. At present, the publicity of Pear Video has achieved results. In the future development, the construction of self-owned platform should become the main task, and only in this way can the development of platform be improved. First of all, Pear Video should explore more business models and build an ecological platform. At present, the development of short video industry is in full bloom, and there is no industry giant. The phenomenon of homogenization of short video platforms is not prominent, and each short video platform is mining different contents in the vertical field. Better developed short 
video platforms, such as second shot and beautiful shot, rely on various ecosystems such as Ali, Tencent and Weibo to gain more development opportunities and platform hits. Pear Video belongs to a single short video platform at present, which has no relying ecosystem, and its platform click through rate can only be obtained by its own content and operation. In the construction of the future platform, Pear Video can learn the application operation modes such as second shot and beautiful shot, and constantly make large financial support to find a strong ecosystem that can be respected through high-quality content. It is necessary to enhance the development potential of capital and operation through the construction of ecological platform, and obtain a new round of dividends for the development of short video industry. At the same time, increase the number and traffic of platform users with the influence of ecosystem. In addition, Pear Video should pay attention to the operation of content copyright and the extension of industrial chain. Content copyright operation is mainly to achieve copyright cooperation with external platforms by means of full copyright IP shareholding and joint investment. Pear Video can be sold by copyright to foreign short video platforms, extending the industrial chain of its own information short video, tapping deeper development potential to obtain financial support and build its own platform.

Secondly, strengthen the community-based operation of the platform and use the community to promote it. Community is a group with common social attributes, and community users have common preferences and values. Pear Video platform, through high-quality content and community construction, makes the audience interact with each other, thus creating a sense of belonging and identity. Through the social process on the platform, a common value orientation is formed and condensed into a community. Once the community comes into being, it will help to provide information feedback for Pear Videos and enhance the interactive relationship with the audience. If Pear Video wants to gain the ability to enhance its platform click-through rate acquisition and reduce its dependence on external platforms, it needs to make the audience community generate motivation and conduct community-based operation. The purpose of community operation is to stimulate community vitality. Attract more platform traffic through the boutique audience. Community-based operation should use community thinking, that is, from the user's point of view, dig deep into human nature. Pear Video should arouse the audience's thinking and make community interact through valuable content. By subdividing the community members, the integration of the members can be improved. Conduct regular activities to promote the development of community relations and build relationship trust through interaction. Use the influence of audience community to push customers, spread content and put advertisements on the platform, tap potential users and enhance platform click-through rate.

Finally, the platform page setup is simplified. Short video users use the platform more by fragmentation time, which requires higher simplicity in platform operation. If the platform design is too complex, the target users will have less favorable impression on the platform because of its complex functions, and the platform design will be simplified. It is beneficial to users' operation, lowers the threshold of users' contact with information, and can improve the user experience of the platform. This will encourage users to be more willing to watch short-sighted information on the platform, instead of clicking on videos on social media. Pages are sorted by time or topic heat, which simplifies the interface of the platform and improves the dissemination effect of short information videos.

\section{Conclusion}

Although the development of short information video in China is limited by the length of time, the content of short video will be less than that of traditional news, but with the development of mobile technology, short information video can not be ignored in the future news development. Pear Video, as the earliest information short video application in China, has attracted users' attention as soon as it was launched. The competitiveness of Pear Video lies in its content creativity, from entertainment to information.

\section{References}

1. Wang N. Research on the communication mechanism of information short video-Taking Pear Video as an example (in Chinese). Audiovisual 2020; (7): 155-156. 
2. Guan C. Research on the profit of information short video platform-Taking Pear Video as an example (in Chinese). Journal of News Research 2020; 11(8): 197-198.

3. Fu H. Media research of information short video-Taking Pear Video as an example (in Chinese). News Communication 2020; (8): 23-25.

4. Han X. Production mode and development strategy of information short videos (in Chinese). Sound Screen World 2020; (8): 98-99.

5. Yi R. The innovation and reflection of short video news on news information dissemination-Taking Pear Video as an example (in Chinese). Audiovisual 2020; (4): 170-171.

6. Zhang Y. The layout, trend and prospect of news short videos in China (in Chinese). News World 2020 ; (3): $62-65$.

7. Yang X. Innovation path of information short video under 5g background (in Chinese). Young Journalists 2020; (5): 80-81.

8. Wang X, Li C. Research on the communication strategy of information short video-Taking Pear Video as an example (in Chinese). Sound Screen World 2019; (12): 84-88.

9. Hu L. Dilemma and path of short video platform of news information-Taking Pear Video as an example (in Chinese). Audiovisual Society 2019; (5): 45-50.

10. Ren D. Breakthrough and advancement of information short videos (in Chinese). News Front 2019; (11): $26-29$. 